Rikke S. Møller, $\mathrm{PhD*}$

Thomas V. Wuttke, MD*

Ingo Helbig, MD

Carla Marini, MD, PhD

Katrine M. Johannesen, MD

Eva H. Brilstra, MD

Ulvi Vaher, MD

Ingo Borggraefe, MD

Inga Talvik, MD

Tiina Talvik, MD

Gerhard Kluger, MD

Laurence L. Francois, MD

Gaetan Lesca, MD, PhD

Julitta de Bellescize, MD

Susanne Blichfeldt, MD

Nicolas Chatron, MD

Nils Holert, MD

Julia Jacobs, MD

Marielle Swinkels, MD

Cornelia Betzler, MD

Steffen Syrbe, MD

Marina Nikanorova, MD

Candace T. Myers, PhD

Line H.G. Larsen, MSc

Sabina Vejzovic, MSc

Manuela Pendziwiat, MSc

Sarah von Spiczak, MD

Sarah Hopkins, MD

Holly Dubbs, MSc

Yuan Mang, MSc

Konstantin Mukhin, MD

Hans Holthausen, MD

Koen L. van Gassen, PhD

Hans A. Dahl, PhD

Niels Tommerup, MD, PhD

Heather C. Mefford, MD, $\mathrm{PhD}$

Guido Rubboli, MD

Renzo Guerrini, MD

Johannes R. Lemke, MD

Holger Lerche, MD

Hiltrud Muhle, MD $\ddagger$

Snezana Maljevic, $\mathrm{PhD} \ddagger$

Correspondence to

Dr. Møller:

rimo@filadelfia.dk

\title{
Mutations in GABRB3
}

\section{From febrile seizures to epileptic encephalopathies}

\section{ABSTRACT}

Objective: To examine the role of mutations in GABRB3 encoding the $\beta_{3}$ subunit of the GABA receptor in individual patients with epilepsy with regard to causality, the spectrum of genetic variants, their pathophysiology, and associated phenotypes.

Methods: We performed massive parallel sequencing of GABRB3 in 416 patients with a range of epileptic encephalopathies and childhood-onset epilepsies and recruited additional patients with epilepsy with GABRB3 mutations from other research and diagnostic programs.

Results: We identified 22 patients with heterozygous mutations in GABRB3, including 3 probands from multiplex families. The phenotypic spectrum of the mutation carriers ranged from simple febrile seizures, genetic epilepsies with febrile seizures plus, and epilepsy with myoclonic-atonic seizures to West syndrome and other types of severe, early-onset epileptic encephalopathies. Electrophysiologic analysis of 7 mutations in Xenopus laevis oocytes, using coexpression of wild-type or mutant $\beta_{3}$, together with $\alpha_{5}$ and $\gamma_{2 s}$ subunits and an automated 2-microelectrode voltage-clamp system, revealed reduced GABA-induced current amplitudes or GABA sensitivity for 5 of 7 mutations.

Conclusions: Our results indicate that GABRB3 mutations are associated with a broad phenotypic spectrum of epilepsies and that reduced receptor function causing GABAergic disinhibition represents the relevant disease mechanism. Neurology ${ }^{\circledR}$ 2017;88:483-492

\section{GLOSSARY}

DS = Dravet syndrome; $\mathbf{E E}=$ epileptic encephalopathies; $\mathbf{E O A E}=$ early-onset absence epilepsy; ExAC = Exome Aggregation Consortium; FS = febrile seizures; GFS + = genetic epilepsies with febrile seizures plus; $\mathbf{G G E}=$ genetic generalized epilepsies; ID = intellectual disability; LGS = Lennox-Gastaut syndrome; MAE = epilepsy with myoclonic atonic seizures; WS $=$ West syndrome; $\mathbf{W} \mathbf{T}=$ wild-type

Disruption of GABAergic inhibition has been shown to cause epileptic seizures. ${ }^{1} \mathrm{GABA}_{\mathrm{A}}$ receptors are ligand-gated anion channels, and mutations in genes encoding different receptor subunits, e.g., GABRG2, GABRA1, GABRD, GABRB2, and GABRB3, have been associated with a wide spectrum of epilepsies from mild genetic generalized epilepsies (GGE) ${ }^{2-6}$ to epileptic encephalopathies (EE). ${ }^{7-10}$

*These authors contributed equally to this work as first authors.

$¥$ These authors contributed equally to this work as last authors.

From the Danish Epilepsy Centre (R.S.M., K.M.J., M.N.), Dianalund; Institute for Regional Health Services (R.S.M., K.M.J., M.N.), University of Southern Denmark, Odense; Department of Neurology and Epileptology (T.V.W., S.V., H.L., S.M.), Hertie Institute for Clinical Brain Research, and Department of Neurosurgery (T.V.W.), University of Tübingen; Department of Neuropediatrics (I.H., M.P., S.v.S., H.M.), University Medical Center Schleswig-Holstein, Kiel, Germany; Division of Neurology (I.H., S.H., H.D.), The Children's Hospital of Philadelphia, PA; Neuroscience Department (C.M., R.G.), Children's Hospital Anna Meyer-University of Florence, Italy; Department of Genetics (E.H.B., M.S., K.L.v.G.), University Medical Center Utrecht, the Netherlands; Department of Neurology and Neurorehabilitation (U.V., I.T., T.T.), Children's Clinic of Tartu University Hospital, Estonia; Department of Pediatric Neurology and Epilepsy Center (I.B.), LMU Munich, Germany; Department of Pediatrics (I.T., T.T.), University of Tartu; Tallinn Children's Hospital (I.T.), Tallinn, Estonia; Clinic for Neuropediatrics and Neurorehabilitation (G.K., C.B., H.H.), Epilepsy Center for Children and Adolescents, Schön Klinik Vogtareuth, Germany; Paracelsus Medical Private University (G.K.), Salzburg, Austria; Neuropeadiatric Department (L.L.F.), Hospices Civils de Lyon; Department of Genetics (G.L., N.C.), Lyon University Hospitals; Claude Bernard Lyon I University (G.L., N.C.); Lyon Neuroscience Research Centre (G.L., N.C.), CNRS UMR5292, INSERM U1028; Epilepsy, Sleep and Pediatric Neurophysiology Department (J.d.B.), Lyon University Hospitals, France; Clinic for Pediatric Neurology (S.B.), Pediatric Department, University Hospital, Herlev, Denmark; Kleinwachau (N.H.), Sächsisches Epilepsiezentrum Radeberg, Dresden; Department of Neuropediatrics/Epilepsy Center (J.J.), University Medical Center Freiburg; Department of General Paediatrics (S.S.), Division of Child Neurology and Inherited Metabolic Diseases, Centre for Paediatrics and Adolescent Medicine, University Hospital Heidelberg; Department of Women and Child Health (S.S.), Hospital for Children and Adolescents, University of Leipzig Hospitals and Clinics, Germany; Department of Pediatrics (C.T.M., H.C.M.), Division of Genetic Medicine, University of Washington, Seattle; Amplexa Genetics (L.H.G.L., H.A.D.), Odense, Denmark; Northern German Epilepsy Center for Children and Adolescents (S.v.S.), Schwentinental-Raisdorf, Germany; Wilhelm Johannsen Centre for Functional Genome Research (Y.M., N.T.), Department of Cellular and Molecular Medicine, University of Copenhagen; Danish Epilepsy Center (G.R.), Filadelfia/University of Copenhagen, Denmark;

Department of Diagnostics (J.R.L.), Institute of Human Genetics, University of Leipzig; and Svt. Luka's Institute of Child Neurology and Epilepsy (K.M.), Moscow, Russia. Dr Maljevic is currently at the Florey Institute of Neuroscience and Mental Health, Melbourne, Australia.

Go to Neurology.org for full disclosures. Funding information and disclosures deemed relevant by the authors, if any, are provided at the end of the article. 
Mutations in $G A B R B 3$ encoding the $\beta_{3}$ subunit of the $\mathrm{GABA}_{\mathrm{A}}$ receptor have recently been reported in patients with severe epilepsies through large-scale studies..$^{7,911-14}$ However, the specific role of $G A B R B 3$ pathogenic variants in the disease context has only recently been assessed in a single study for a small number of mutations, ${ }^{15}$ and data on the functional consequences of most of the mutations are still lacking.

Here, we systematically analyze a large cohort of patients with various epilepsies for mutations in GABRB3 and integrate clinical and genetic data from additional, unreported patients. We provide insight into the mutational landscape of $G A B R B 3$, including several recurrent mutations, and characterize the functional consequences of mutations for milder and more severe phenotypes using 2-microelectrode voltage clamping in Xenopus laevis oocytes.

METHODS Patients. We screened a cohort of 416 patients sequentially referred for testing with various childhood-onset epilepsies for mutations in the GABRB3 gene using a nextgeneration sequencing panel. Genomic DNA from blood was extracted with standard methods, and a next-generation sequencing panel screening method was applied that was based on the Ion Torrent PGM platform. Ion AmpliSeq (kit version 2.0) or Sureselect library building methods were used with subsequent clonal amplification and enrichment on an Ion OneTouch 2 system with the Ion PGM Template OT2 200 Kit, followed by sequencing on the Ion Torrent system with the Ion PGM 200 Sequencing Kit. In parallel, we ascertained additional, previously unreported patients through centers in Europe and the United States. The probands and their families underwent detailed clinical examinations, review of the medical files, MRIs, and EEG investigations. Seizures were diagnosed according to the International League Against Epilepsy, and epilepsy syndromes were established when possible. ${ }^{16}$

Mutation analysis. In 2 cases, mutations were identified with the gene panel mentioned above, which included targeted capture of all exons and at least 5 base pairs of flanking intronic sequence of GABRB3.

Patients with $G A B R B 3$ mutations ascertained through collaborators were diagnosed through established diagnostic programs or research studies. Sanger sequencing was used to confirm all mutations and to perform segregation analysis.

Standard protocol approvals, registrations, and patient consents. The study was approved by the local ethics committees. All probands or, in case of minors, their parents or legal guardians gave informed consent.

Functional data. Mutagenesis and RNA preparation. We used the Quick Change kit (Stratagene, La Jolla, CA) to engineer 7 variants (p.V37G, p.R111*, p.T157M, p.Y184H, p.L256Q, p.Y302C, and p.R429Q) in $\mathrm{CDNA}$ encoding the $\mathrm{GABA}_{\mathrm{A}}$ receptor subunit $\beta_{3}$ (NM_000814.4, Origene Technologies, Rockville, $\mathrm{MD})$. cRNAs were prepared with a custom laboratory protocol or the T7 mMessage mMachine kit from Ambion (Thermo Fisher Scientific, Waltham, MA).

Oocyte preparation and injection. The use of animals and all experimental procedures were approved by local authorities (Regierungspraesidium Tuebingen, Tuebingen, Germany). Oocytes were obtained from the Institute of Physiology I, Tuebingen and prepared as previously described..$^{10}$ Briefly, the procedure included treatment with collagenase $(1 \mathrm{mg} / \mathrm{mL}$ of type CLS II collagenase, Biochrom KG, Berlin, Germany) in OR-2 solution (mmol/L: $82.5 \mathrm{NaCl}, 2.5 \mathrm{KCl}, 1 \mathrm{MgCl}_{2}$, and 5 Hepes, $\mathrm{pH}$ 7.6), followed by thorough washing and storing at $16^{\circ} \mathrm{C}$ in Barth solution [mmol/L: $88 \mathrm{NaCl}, 2.4 \mathrm{NaHCO}_{3}, 1 \mathrm{KCl}, 0.33$ $\mathrm{Ca}\left(\mathrm{NO}_{3}\right)_{2}, 0.41 \mathrm{CaCl}_{2}, 0.82 \mathrm{MgSO}_{4}$, and 5 Tris/ $\mathrm{HCl}, \mathrm{pH} 7.4$ with $\mathrm{NaOH}$ ] supplemented with $50 \mu \mathrm{g} / \mathrm{mL}$ gentamicin (Biochrom KG, Berlin, Germany). Equivalent amounts of cRNA were injected in parallel in oocytes from the same batch, plated in 96-well plates, and recorded at day 3 after injection. ${ }^{10,17}$ The subunit combination was $\alpha_{5}, \beta_{3}$, and $\gamma_{2}$ in a 1:1:2 ratio. All cRNA concentrations were adjusted to $2 \mu \mathrm{g} / \mu \mathrm{L}$ and $70 \mathrm{~nL}$ of the corresponding cRNA mixtures injected with the Robooinject system (Multi Channel Systems, Reutlingen, Germany).

Automated oocyte 2-microelectrode voltage clamp. $\mathrm{GABA}_{\mathrm{A}}$ receptor-mediated currents were recorded in oocytes at room temperature $\left(20^{\circ} \mathrm{C}-22^{\circ} \mathrm{C}\right)$ with the Roboocyte 2 system (Multi Channel Systems). Intracellular glass microelectrodes had a resistance of 0.3 to $1 \mathrm{M} \Omega$ when filled with $1 \mathrm{~mol} / \mathrm{L} \mathrm{KCl} / 1.5 \mathrm{~mol} / \mathrm{L}$ K-acetate. ND96 (mmol/L: $93.5 \mathrm{NaCl}, 2 \mathrm{KCl}, 1.8 \mathrm{CaCl}_{2}, 2$ $\mathrm{MgCl}_{2}$, and 5 Hepes, $\mathrm{pH} 7.5$ ) was used as the bath solution. Currents were sampled at $1 \mathrm{kHz}$, and the holding membrane potential was $-70 \mathrm{mV} .{ }^{10}$

Electrophysiologic data analysis. Currents were analyzed with Roboocyte2+ (Multi Channel Systems), Microsoft Excel (Microsoft, Redmond, WA), and GraphPad Prism software (GraphPad Software, La Jolla, CA). Current amplitudes in response to $1 \mathrm{mmol} / \mathrm{L}$ GABA recorded on the same day were normalized to the mean value of the wild-type (WT) so that normalized data from different experiments could be pooled together. To obtain dose-response curves, the current response to each GABA concentration was normalized to the maximum response evoked by the highest GABA concentration $(1 \mathrm{mmol} / \mathrm{L})$, plotted against the respective concentration and for each cell fit to the following 4-parameter equation:

$$
\mathrm{Y}(\mathrm{X})=\mathrm{B}_{\max } \times \mathrm{X}^{\mathrm{h}} /\left(\mathrm{K}_{\mathrm{d}}^{\mathrm{h}}+\mathrm{X}^{\mathrm{h}}\right)
$$

where $B_{\max }$ is the maximum response to GABA, $K_{d}$ is the concentration to achieve half-maximum response, and $h$ is the Hill slope.

Data and statistical analyses. Data were tested for normal distribution with GraphPad Prism 6. Groups were compared with the use of one-way analysis of variance with the Tukey post hoc test. All data are presented as mean \pm SEM.

RESULTS Mutation analysis. In our screening cohort of 416 patients, we identified 2 patients $(0.4 \%)$ with de novo GABRB3 mutations and ascertained 20 additional patients through collaborators, yielding in total 22 patients with presumed pathogenic variants in GABRB3 (table 1). Three patients have previously been reported (patients 3, 12, and 13). ${ }^{9} 18$ Eighteen of 22 mutations were missense; 3 of 22 were truncating mutations; and one alteration was a partial duplication of exons 1 through 9 . 


\begin{tabular}{|c|c|c|c|c|c|c|c|c|c|}
\hline Patient & $\begin{array}{l}\text { Epilepsy } \\
\text { syndrome }\end{array}$ & $\begin{array}{l}\text { Seizure } \\
\text { onset }\end{array}$ & Seizure types/fsens & $\begin{array}{l}\text { Seizure outcomel } \\
\text { age at offset }\end{array}$ & $\begin{array}{l}\text { Intellectual } \\
\text { disability }\end{array}$ & EEG & Additional features & MRI & $\begin{array}{l}\text { cDNA*/protein position detected } \\
\text { by (1), (2) }\end{array}$ \\
\hline \multicolumn{10}{|c|}{$\begin{array}{l}\text { Familial epilepsies with } \\
\text { febrile and generalized } \\
\text { seizures }(n=2)\end{array}$} \\
\hline 1 (female) & $\mathrm{EOAE}^{\mathrm{a}}$ Fam1 & $9 \mathrm{mo}$ & FS, A, GTCS, fsens+ & Sz-free/4 y & Normal intellect & GSW, PSW & None & None & c.110T>G, p.V37G, paternal, (1) \\
\hline 2 (male) & GEFS,$+{ }^{a}$ Fam2 & $6 \mathrm{mo}$ & $\begin{array}{l}\text { FS, dyscognitive, GTCS, } \\
\text { fsens }+\end{array}$ & Sz-free/10 y & Normal intellect & PSW, R frontal & None & Normal & c.1286G >A, p.R429Q, maternal, (1) \\
\hline \multicolumn{10}{|c|}{$\begin{array}{l}\text { Focal/multifocal/ } \\
\text { unclassifiable } \\
\text { epilepsies }(n=4)\end{array}$} \\
\hline 3 (male) & Focal epilepsy & $7 \mathrm{mo}$ & Focal, unclassified, fsens + & Rare Sz & $\begin{array}{l}\text { Mild, plateau at } \\
7 \mathrm{mo}\end{array}$ & Focal IED & Mild ataxia, hypotonia & Normal & c.905A>G, p.Y302C, de novo (2) \\
\hline 4 (female) & Focal epilepsy & $15 \mathrm{mo}$ & $\begin{array}{l}\text { Focal clonic, focal, } \\
\text { myoclonic, fsens }+\end{array}$ & Sz-free/2 y & Mild & $\begin{array}{l}\text { Multifocal, focal } \\
\text { IED, +diffuse SW } \\
\text { activated by sleep }\end{array}$ & Ataxia, hypotonia & Normal & c.902C > T, p.P301L, de novo (2) \\
\hline 5 (female) & Unclassified & $4 \mathrm{mo}$ & $\begin{array}{l}\text { SE, clonic, hypomotor, } \\
\text { GTCS, fsens- }\end{array}$ & Sz-free/3 y & Moderate & Multifocal & Autistic features & $\begin{array}{l}\text { Bifrontal } \\
\text { heterotopia }\end{array}$ & c.695G >A, p.R232Q, de novo (1) \\
\hline 6 (male) & Unclassified & $11 \mathrm{mo}$ & A, EM, unclassified, fsens + & $\begin{array}{l}\text { Sz-free for } 2 \text { yl } \\
\text { rare seizures }\end{array}$ & Moderate & Multifocal & None & Normal & c.695G >A, p.R232Q, de novo (2) \\
\hline \multicolumn{10}{|c|}{$\begin{array}{l}\text { EE with mild to severe } \\
\text { intellectual disability } \\
(\mathrm{n}=16)\end{array}$} \\
\hline 7 (male) & MAE & $36 \mathrm{mo}$ & M, MA, GTCS, fsens- & Sz-free/4 y & $\begin{array}{l}\text { Moderate, plateau } \\
\text { at } 3 y\end{array}$ & GSW, PSW & None & Normal & c.8delG, p.Gly3fs*26, unknown (1) \\
\hline 8 (male) & MAE & $9 \mathrm{mo}$ & FS, GTCS, MA, fsens + & Daily Sz & $\begin{array}{l}\text { Mild, stagnation at } \\
16 \mathrm{mo}\end{array}$ & GSW & Mild ataxia & Normal & c.227C>G, p.S76C, de novo (1) \\
\hline 9 (male) & MAE & $12 \mathrm{mo}$ & FS, MA, GTCS, fsens+ & Sz-free/4 y & $\begin{array}{l}\text { Severe, } 24 \text { mo } \\
\text { regression }\end{array}$ & Multifocal & Autism & Normal & c.331C>T, p.R111*, maternal (1) \\
\hline 10 (female) & MAE & $12 \mathrm{mo}$ & MA, fsens + & Sz-free/18 mo & Mild & PSW & $\begin{array}{l}\text { Behavioral issues } \\
\text { (aggression) }\end{array}$ & Normal & $\begin{array}{l}\text { c.425G }>\text { T, p.R142L, maternal } \\
\text { (mosaic: } 10 \%-20 \% \text { ) (1) }\end{array}$ \\
\hline 11 (male) & MAE & $14 \mathrm{mo}$ & MA, GTCS, fsens+ & GTCS in clusters & Mild & GSW & Normal & Normal & c.550T>C, p.Y184H, de novo (1) \\
\hline 12 (male) & DS-like, ${ }^{a}$ Fam3 & $8 \mathrm{mo}$ & FS, GTCS, Abs, M, A, fsens+ & Weekly Sz & Mild, delays at $2.5 \mathrm{y}$ & Bilateral SW & ADHD & Normal & c.470C>T, p.T157M, maternal, (2) \\
\hline 13 (male) & $\mathrm{EE} / \mathrm{WS}$ & $1 \mathrm{~d}$ & Focal, GTCS, IS, tonic, fsens- & Daily Sz & $\begin{array}{l}\text { Severe, } 3 \text { mo } \\
\text { stagnation }\end{array}$ & $\begin{array}{l}\text { Hypsarrhythmia, } \\
\text { GSSW }\end{array}$ & Hypotonia, dyskinesia & Hypomyelination & c.767T>A, p.L256Q de novo (1) \\
\hline 14 (male) & EE/WS & $4 \mathrm{mo}$ & $\begin{array}{l}\text { Dyscognitive, clonic, } \\
\text { IS, fsens- }\end{array}$ & Sz-free/1 y & Moderate & $\begin{array}{l}\text { Multifocal, burst } \\
\text { sup }\end{array}$ & Hypotonia & Hypomyelination & Exon 1-9 duplication, de novo (1) \\
\hline 15 (male) & EE/WS & $8 \mathrm{mo}$ & IS, tonic, FS, GTCS, fsens- & Rare $\mathrm{Sz}$ & $\begin{array}{l}\text { Moderate, } \\
\text { regression (12 m) }\end{array}$ & Multifocal & Autism, ataxia, tremor & Normal & $\begin{array}{l}\text { c.205G > A, p.A69T, transcript: } \\
\text { ENST00000541819.2 unknown (1) }\end{array}$ \\
\hline 16 (female) & EE/LGS & $17 \mathrm{mo}$ & $\begin{array}{l}\text { A, tonic, dyscognitive, } \\
\text { GTCS, fsens- }\end{array}$ & Frequent Sz & Severe & Multifocal & $\begin{array}{l}\text { Strabismus, } \\
\text { hyperactivity and } \\
\text { aggression }\end{array}$ & Normal & c.905A>G, p.Y302C, de novo (1) \\
\hline 17 (female) & EOEE & $2.5 \mathrm{mo}$ & $\begin{array}{l}\text { Focal, clonic, migrating, } \\
\text { fsens- }\end{array}$ & Weekly Sz & Severe & Multifocal & $\begin{array}{l}\text { Acquired microcephaly } \\
\text { (HC }<-2 \text { SD), } \\
\text { hypotonia, quadriplegia }\end{array}$ & $\begin{array}{l}\text { Severe, diffuse } \\
\text { brain atrophy }\end{array}$ & c.372A>C, p.L124F, de novo (1) \\
\hline
\end{tabular}


Table 1 Continued

\begin{tabular}{|c|c|c|c|c|c|c|c|c|c|}
\hline Patient & $\begin{array}{l}\text { Epilepsy } \\
\text { syndrome }\end{array}$ & $\begin{array}{l}\text { Seizure } \\
\text { onset }\end{array}$ & Seizure types/fsens & $\begin{array}{l}\text { Seizure outcomel } \\
\text { age at offset }\end{array}$ & $\begin{array}{l}\text { Intellectual } \\
\text { disability }\end{array}$ & EEG & Additional features & MRI & $\begin{array}{l}\text { cDNA*/protein position detected } \\
\text { by (1), (2) }\end{array}$ \\
\hline 18 (male) & EOEE & $3 \mathrm{mo}$ & Focal, tonic, fsens- & Rare tonic $\mathrm{Sz}$ & Severe & Multifocal & $\begin{array}{l}\text { Acquired microcephaly } \\
\text { (HC }<-3 \mathrm{SD}) \\
\text { quadriplegia }\end{array}$ & $\begin{array}{l}\text { Hypoplastic } \\
\text { cerebellum }\end{array}$ & c.761C $>$ T, p.S254F, de novo (1) \\
\hline 19 (female) & EOEE & $2.5 \mathrm{mo}$ & Hypomotor, M, fsens+ & Rare Sz & Severe & Multifocal & Hypotonia & $\begin{array}{l}\text { Atrophy, } \\
\text { hypomyelination, } \\
\text { thin CC }\end{array}$ & c.554C >T, p.T185I, de novo (2) \\
\hline 20 (male) & EOEE & $5 \mathrm{mo}$ & A, tonic, SE, fsens NA & Weekly Sz & Severe & Multifocal & Hypotonia & $\begin{array}{l}\text { Volume loss, } \\
\text { brainstem } \\
\text { atrophy }\end{array}$ & c.380A $>$ G, p.K127R, de novo (1) \\
\hline 21 (male) & $\begin{array}{l}\text { Focal epilepsyl } \\
\text { EE }\end{array}$ & $11 \mathrm{mo}$ & $\begin{array}{l}\text { Dyscognitive, GTCS, } \\
\text { unclassified, A, fsens NA }\end{array}$ & $\begin{array}{l}\text { Frequent } \mathrm{Sz} \text { up to } \\
300 \mathrm{Sz} / \mathrm{d}\end{array}$ & Severe & Multifocal & $\begin{array}{l}\text { Autistic features, hand } \\
\text { stereotypies, Rett-like }\end{array}$ & Normal & $\begin{array}{l}\text { c. } 758 \text { C > T, p.P253L, (mosaic: 20\%), } \\
\text { de novo (1) }\end{array}$ \\
\hline 22 (male) & EE/LGS & $11 \mathrm{mo}$ & $\begin{array}{l}\text { Febrile GTCS, dyscognitive, } \\
\text { Abs-like, tonic, fsens NA }\end{array}$ & Daily & $\begin{array}{l}\text { Severe, regression } \\
(5 \mathrm{y})\end{array}$ & Multifocal & $\begin{array}{l}\text { Self-injury episodes, } \\
\text { hand stereotypies, } \\
\text { hyperventilation } \\
\text { episodes }\end{array}$ & Normal & c.694C>T, pArg232*, paternal (1) \\
\hline \multicolumn{10}{|l|}{$\begin{array}{l}\text { Family members with } \\
\text { GABRB3 mutations } \\
(\mathrm{n}=9)\end{array}$} \\
\hline Fam1, I.2 (male) & $\mathrm{FS}+$ & $\begin{array}{l}<5 \mathrm{y} \\
14 \mathrm{y}\end{array}$ & FS, GTCS & $\begin{array}{l}\text { Multiple FSs, } \\
\text { single GTCS Sz- } \\
\text { free }\end{array}$ & Normal intellect & NA & None & Not done & c.110T>G, p.V37G, paternal \\
\hline Fam1, II.2 (female) & FS & $9 \mathrm{mo}$ & FS & $6 \mathrm{FS}, \mathrm{Sz}$-free & Normal intellect & NA & None & Not done & c.110T>G, p.V37G, paternal \\
\hline Fam2, I.1 (female) & No $\mathrm{Sz}$ & - & - & No $\mathrm{Sz}$ & Normal intellect & NA & None & Not done & c.1286G >A, p.R429Q, maternal \\
\hline Fam2, II.2 (male) & $\mathrm{FS}+$ & $2 y, 5 y$ & FS, GTCS & Sz-free & Normal intellect & $\begin{array}{l}\text { Occipital sharp- } \\
\text { slow waves }(9 \text { y) }\end{array}$ & None & Not done & c.1286G $>$ A, p.R429Q, maternal \\
\hline Fam3, I.1 (female) & FS & NA & FS & $\begin{array}{l}\text { Multiple FSs, Sz- } \\
\text { free }\end{array}$ & Normal intellect & NA & None & Not done & c. $470 \mathrm{C}>\mathrm{T}$, p.T157M, \\
\hline Fam3, II.2 (female) & GEFS+ & $\begin{array}{l}\text { First } \\
\text { year of } \\
\text { life }\end{array}$ & FS, GTCS, Abs & Sz-free & Normal intellect & NA & None & Not done & c. $470 \mathrm{C}>\mathrm{T}$, p.T157M, maternal \\
\hline Fam3, II.3 (female) & FS & $\begin{array}{l}\text { First } \\
\text { year of } \\
\text { life }\end{array}$ & FS & $\begin{array}{l}\text { Multiple FSs, Sz- } \\
\text { free }\end{array}$ & Normal intellect & NA & None & Not done & c. $470 \mathrm{C}>\mathrm{T}$, p.T157M, maternal \\
\hline Fam3 III.1 (male) & GEFS+ & $\begin{array}{l}\text { First } \\
\text { year of } \\
\text { life }\end{array}$ & FS, GTCS & Sz-free & Normal intellect & Normal & None & Not done & c. $470 \mathrm{C}>\mathrm{T}$, p.T157M, maternal \\
\hline Fam3, III.5 (female) & DS-like & $5 \mathrm{mo}$ & $\begin{array}{l}\text { FS, febrile SE, GTCS, Abs, M, } \\
\text { fsens+ }\end{array}$ & $\begin{array}{l}\text { Weekly to } \\
\text { monthly Sz }\end{array}$ & $\begin{array}{l}\text { Mild delay since age } \\
1.5 \mathrm{y}\end{array}$ & Normal & $\begin{array}{l}\text { Hyperactivity, } \\
\text { aggressiveness }\end{array}$ & Not done & c. $470 \mathrm{C}>\mathrm{T}$, p.T157M, maternal \\
\hline
\end{tabular}

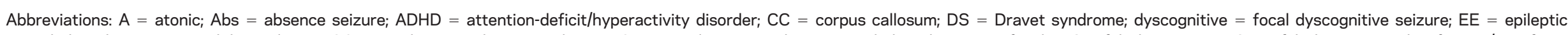

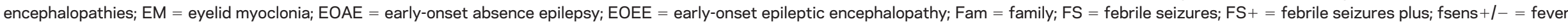

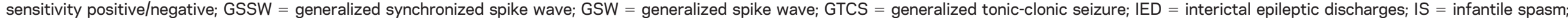

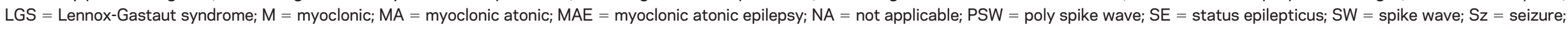
WS $=$ West syndrome; $*$ Transcript $=$ NM_000814.4.

cDNA*/protein position detected by next-generation sequencing panel diagnostic (1) or whole-exome sequencing (2).

${ }^{a}$ Family history (see figure 1B). 
Concerning the inheritance mode, 14 of 22 mutations occurred de novo, including in one patient who was mosaic; 3 of 22 mutations segregated within the family in a dominant fashion; 1 of 22 was inherited from an unaffected mother who was mosaic for the mutation; and 2 of 22 were inherited from an unaffected parent. Complete segregation analysis could not be performed for 2 of 22 mutations, including one recurrent mutation that occurred de novo in 2 other patients in this study.

All missense mutations were predicted to be damaging by 1 or 2 prediction tools (Polyphen 2 or SIFT; table e-1 at Neurology.org). Twenty-one of 22 mutations were not present in the Exome Aggregation Consortium (ExAC) database, whereas one mutation (p.A69T) found in an alternative GABRB3 transcript was observed in 2 of 14,132 chromosomes. The protein positions of the different $G A B R B 3$ mutations are shown in figure $1 \mathrm{~A}$.

Overall mutational landscape. Mining the available literature and databases, we were able to identify 16 additional previously reported $G A B R B 3$ cases (table e-1). ${ }^{7,9,11,12,14,19}$ The majority of the published mutations occurred de novo and were associated with EE. From the complete dataset of 38 patients with GABRB3 mutations, 5 sites with recurring mutations at identical amino acid positions emerged: p.D120N (2x), p.K127R (2x), p.R232Q (2x)/p.R232*(1x), p.Y302C (3x), and p.A305V/p.A305T (1x each) (figure 1A).

Phenotypic analysis. Index patients. The phenotypic spectrum in our study varied from genetic epilepsies with febrile seizures plus (GEFS+) and early-onset absence epilepsy (EOAE) to multifocal epilepsy, EE within the Dravet syndrome (DS) spectrum, epilepsy with myoclonic atonic seizures (MAE), West syndrome (WS), Lennox-Gastaut syndrome (LGS), and other unclassifiable types of EE. The median age at seizure onset was 8.5 months (range 1 day-36 months). Fever-associated seizures were reported in 11 of 19 patients for whom data were available (2 of 2 with GGE, 3 of 4 with unclassified epilepsies, 1 of 1 with DS-like EE, 4 of 5 with MAE, and 1 of 7 with other EE/EOEE).

Sixteen of 22 patients (73\%) had EE, including DS-like EE ( $\mathrm{n}=1)$, MAE $(\mathrm{n}=5)$, WS $(\mathrm{n}=3)$, LGS $(n=2)$, and unclassifiable EE/EOEE $(n=5)$. These patients had various seizure types, including infantile spasms, focal dyscognitive seizures, tonic seizures, myoclonic seizures, atonic seizures, myoclonicatonic seizures, and generalized tonic-clonic seizures. Four of 22 patients had focal or unclassifiable epilepsy with onset between 4 and 15 months and various seizure types. One patient (patient 4) with focal epilepsy had focal EEG abnormalities, which were activated by sleep and became bilaterally diffuse. Three of 4 patients with focal/unclassifiable epilepsy (patients 4-6) became seizure-free between 2 and 3 years of age. However, patient 6 had single seizures after he was tapered off medication. The last patient (patient 3) in this group had rare seizures. Two of 22 patients had generalized epilepsies well controlled on antiepileptic medication, EOAE (patient 1) and GEFS+ (patient 2) with offset at age 4 and 10 years, respectively.

The 2 patients with GGE had normal cognitive skills, in contrast to patients with focal/unclassifiable epilepsy and EE, who presented with mild to severe intellectual disability (ID). Behavioral and psychiatric disturbances, including attention-deficit/hyperactivity disorder, autistic features, agitation, hyperactivity, and aggression, were reported in 8 of the 22 patients (36\%).

Seven of 22 patients (32\%) had MRI abnormalities, including bilateral frontal heterotopia with suspected polymicrogyria (patient 5), hypomyelination (patients 13 and 14), severe diffuse brain atrophy (patient 17), hypoplastic cerebellum (patient 18), generalized volume loss with thin corpus callosum (patient 19 ), or prominent cortical and subcortical volume loss with brainstem atrophy (patient 20). However, no clear pattern typical for GABRB3 mutations emerged from these observations. Except for one patient (patient 5), all MRI abnormalities were detected in patients of the EE subgroup.

Family members. The 2 individuals with GGE (patient $1 /$ family 1 , patient 2 /family 2 ) and the patient with a DS-like phenotype (patient 12/family 3 ; e-supplement) were index patients of multiplex families (table 1 and figure 1B). In total, we identified 9 family members carrying pathogenic $G A B B R 3$ variants, including one unaffected carrier. Seven of the 8 affected family members became seizure-free and had a normal intellect. The sister of patient 12 was classified as DS-like (e-supplement), whereas the remaining affected family members of family 3 had GEFS + or simple FS (table 1). The 2 sibs in family 3 with a DSlike phenotype have previously tested negative for mutations in other DS-associated genes, including $S C N 1 A$, $G A B R G 2$, and GABRA1. Phenocopies presenting with simple FS were observed in each family.

Functional data. To cover the described phenotypic spectrum, a set of 7 mutations was assorted for functional analysis using 2-microelectrode voltage clamp experiments in Xenopus laevis oocytes (figure 2A). The spectrum included mutations associated with familial GEFS+/DS-like (p.R429Q and p.T157M), familial GEFS+ and EOAE (p.V37G), MAE (p.Y184H, p.R111*), WS (p.L256Q), and focal epilepsy/EE/ LGS (p.Y302C).

Recordings of different mutations were performed in parallel with the WT in every batch of oocytes. 
Figure 1 GABRB3 mutations in epilepsy and pedigrees of GABRB3 multiplex families

A

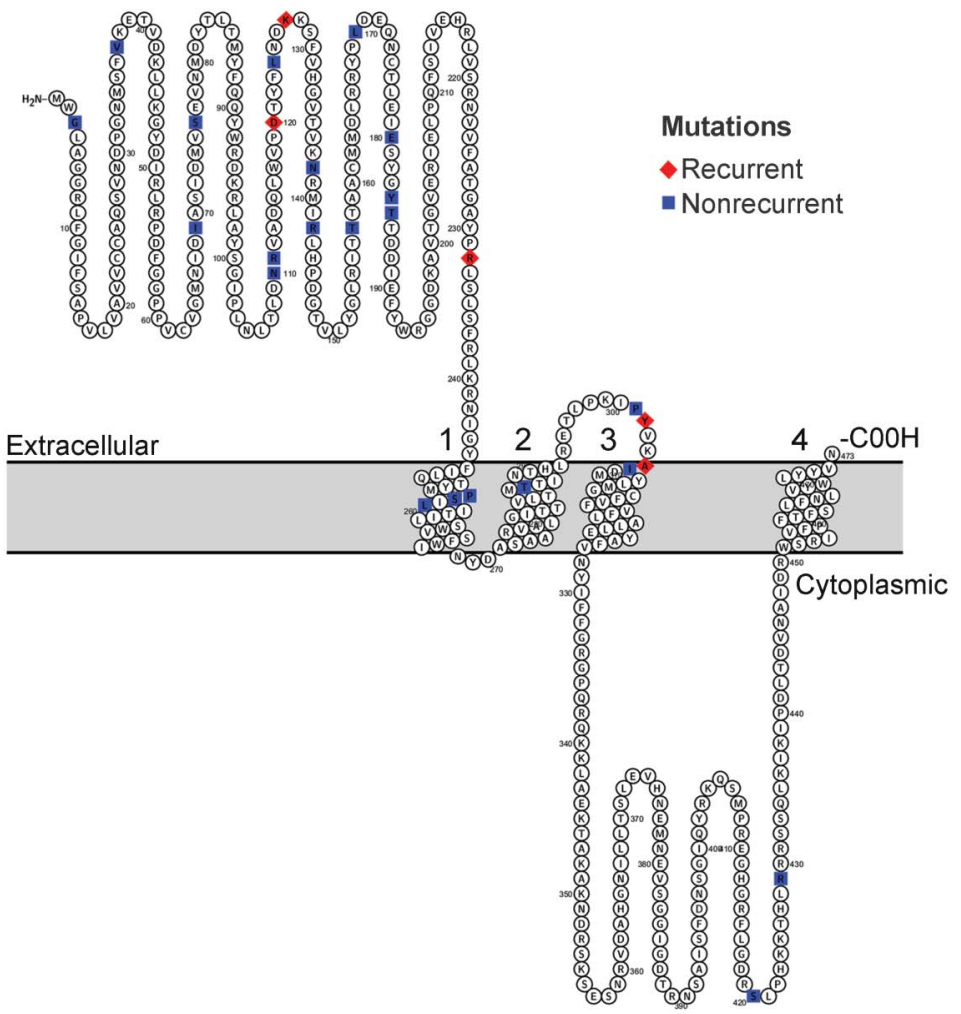

B Family 1: c. $110 \mathrm{~T}>\mathrm{G}$ p.V37G

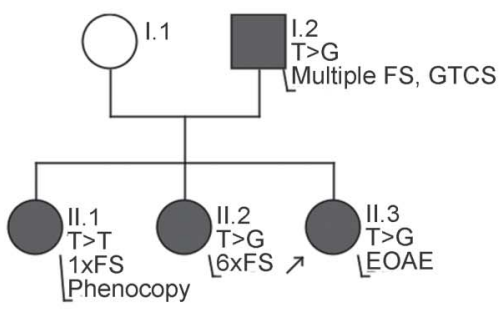

Family 2:

c. 1286 G>A p.R429Q

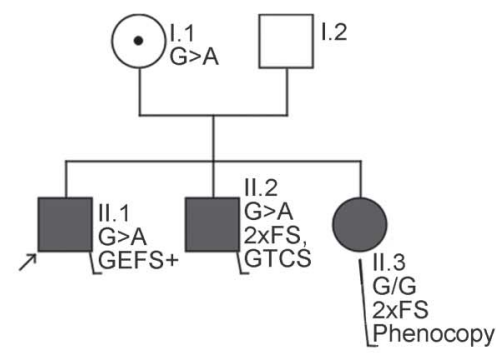

Family 3: c. $470 \mathrm{G}>$ A p.T157M

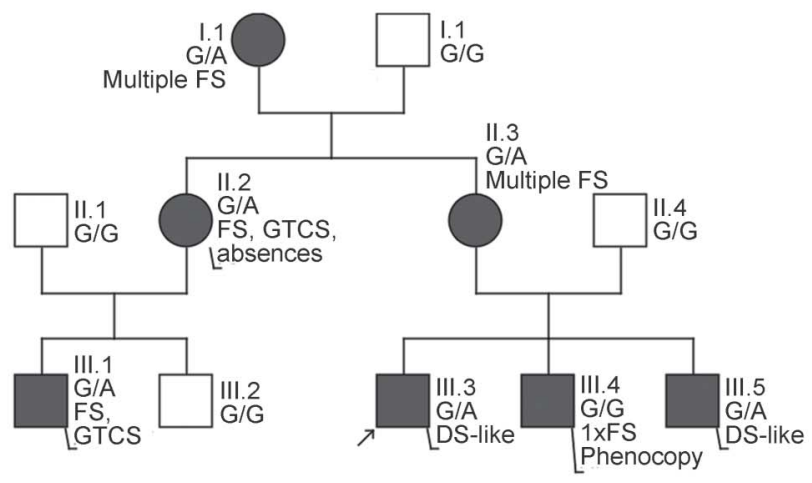

(A) The amino acid locations of the identified GABRB3 missense mutations. (B) Pedigrees of multiplex families carrying GABRB3 mutations. DS = Dravet syndrome; EOAE = early-onset absence epilepsy; FS = febrile seizures; GEFS + = generalized epilepsy with febrile seizures plus; GTCS = generalized tonic-clonic seizure.
Current amplitudes obtained after application of $1 \mathrm{mmol} / \mathrm{L}$ GABA showed tentatively but not significantly smaller currents for p.R429Q and p.T157M. In contrast, strongly and significantly reduced current amplitudes were found for p.V37G, p.Y184H, p.L256Q, p.R111*, and p.Y302C (figure 2B).

To investigate whether a change in the GABA sensitivity of the mutated receptors could be an additional underlying pathophysiologic mechanism, we recorded concentration curves for WT and the mutations with current amplitudes large enough for this type of recording, including p.T157M, the recurring mutation p.Y302C, and p.Y184H. Whereas the dose-response curve obtained for p.T157M revealed no significant difference compared to the WT, the 2 remaining mutations caused a pronounced rightward shift, indicating a significantly reduced GABA sensitivity. This shift appears to explain, at least in part, the pronounced reduction of current amplitudes observed at $1 \mathrm{mmol} / \mathrm{L}$ GABA for the 2 mutations because dose-response curves did not saturate at $1 \mathrm{mmol} / \mathrm{L}$ GABA.

DISCUSSION Even though individual mutations in $G A B R B 3$ have recently been reported in patients with severe epilepsies, ${ }^{7,11,12,14}$ the role of GABRB3 in human epilepsies in terms of causality and associated phenotypes has remained largely unclear. In this study, we describe a comprehensive cohort of patients carrying GABRB3 mutations, delineate the mutational and phenotypic spectrum, and demonstrate loss of protein function as the causative disease mechanism in severe cases.

We found a wide phenotypic spectrum associated with GABRB3 mutations, ranging from FS/GEFS+ and EOAE to unclassified focal epilepsies, MAE, DS-like EE, WS, LGS, and other types of EE. Among the group of patients with EE, the cognitive impairment was more severe and the epilepsy more refractory compared to the group of patients with GEFS+, EOAE, and unclassified focal epilepsy. Within the EE group, patients with MAE had a less severe outcome; 4 of 5 MAE patients became seizure-free, and most of them had mild ID.

We also tested 7 GABRB3 mutations functionally. For 2 mutations (family 2, patient 2: p.R429Q; family 3, patient 12: p.T157M), we did not find significant changes compared to WT receptors. Such a finding is not unusual for the functional screening in Xenopus oocytes, which are not perfectly suited to detect minor abnormalities. Even though the severity of the phenotypes within families was variable, the overall familial phenotype was milder for both mutations compared to patients carrying de novo mutations. Furthermore, the phenotypes are compatible with the known GEFS+ spectrum, including FS, FS+, GEFS+, MAE, and DS, which is also well known from families carrying 

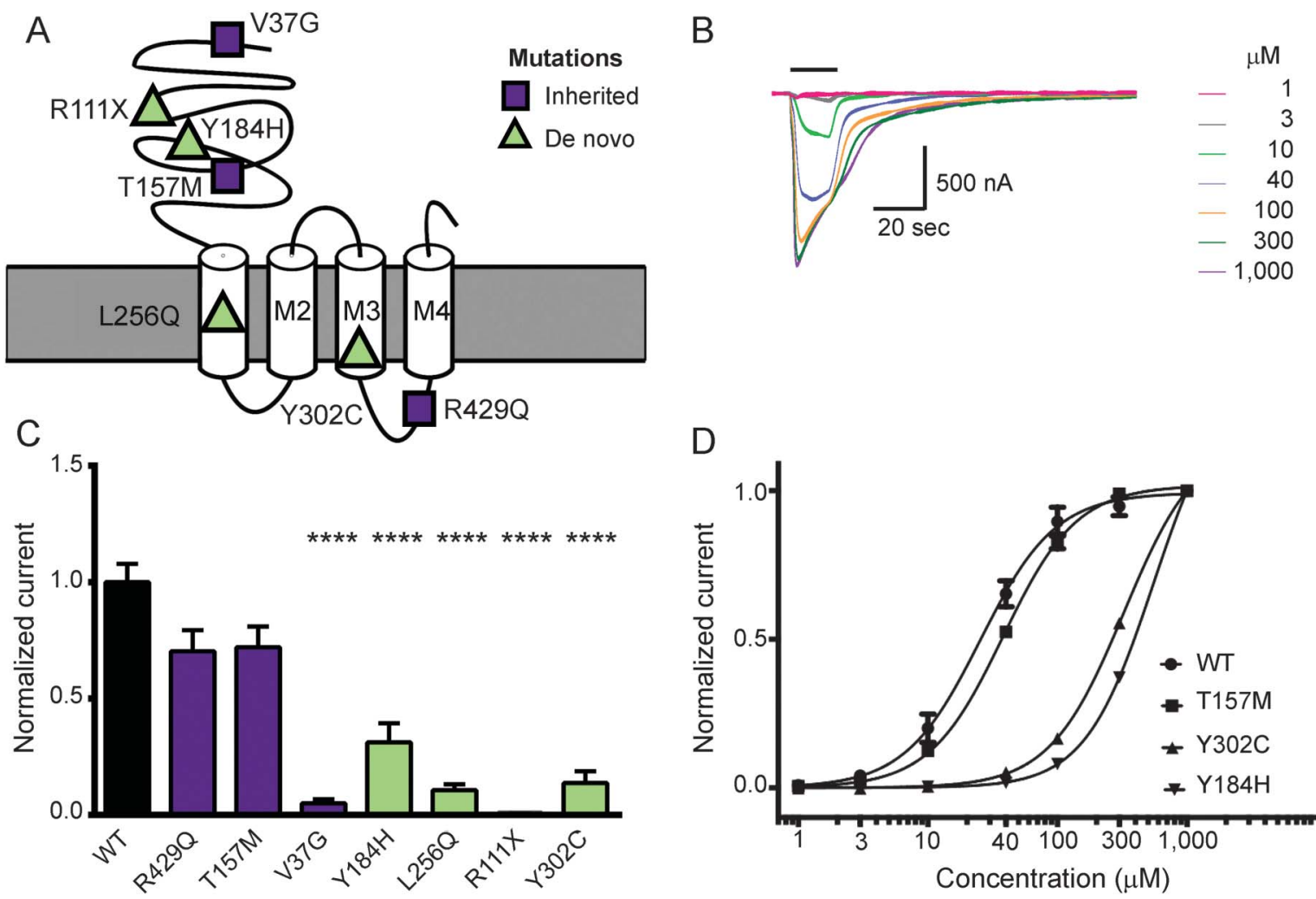

(A) Schematic representation of the $\beta 3$ subunit of the $\mathrm{GABA}_{A}$ receptor, including the predicted positions of the mutated amino acids. (B) Examples of current responses to application of increasing GABA concentrations ( $\mu \mathrm{mol} / \mathrm{L}: 1,3,10,40,100,300$, and 1,000) recorded from Xenopus oocytes expressing wild-type (WT) $\alpha_{5} \beta_{3} \gamma_{2 s}$ receptors. (C) Normalized current response to $1 \mathrm{mmol} / \mathrm{L}$ GABA application for $W T(n=120), R 429 Q(n=41), T 157 M(n=39), V 37 G(n=29), Y 184 H(n=$ 36), L256Q ( $=31)$, R111X ( $=31)$, and Y302C $(n=34)$. **** $<0.0001$, one-way analysis of variance with the Tukey multiple-comparisons test. (D) Doseresponse curve for $\alpha_{5} \beta_{3} \gamma_{2 s}$ WT ( $\left.n=11\right), T 157 \mathrm{M}(n=6), Y 302 \mathrm{C}(\mathrm{n}=3)$, and Y184H $(n=7)$ receptors recorded on application of different GABA concentrations (as in A) and normalized to the maximal response $(1,000 \mu \mathrm{mol} / \mathrm{L})$ for each cell. $E C_{50}$ values were $25.3,38.0,326.4$, and $552.3 \mu \mathrm{mol} / \mathrm{L}$ with the $95 \%$ confidence intervals ranging from 20.7 to $29.9,35.4$ to $40.6,293.8$ to 359.0 , and 433.9 to $670.7 \mu \mathrm{mol} / \mathrm{L}$ for WT, T157M, Y302C, and Y184H, respectively.

mutations in other GEFS+ genes, e.g., SCN1A or GABRG2. ${ }^{20,21}$ Moreover, these mutations are not found in the general population, are evolutionarily conserved, and are predicted to be damaging by bioinformatic programs. In contrast, the third mutation (family 1, patient 1: p.V37G) identified in a family with GGE was found to cause a significant loss-of-function effect. These discrepancies between the genotypes and phenotypes with a severe loss of function in a family with a mild phenotype (family 1) and a virtual lack of GABRB3 impairment in a family with a more severe phenotype (family 3) suggest that factors other than the GABRB3 variant must be contributing to the disease phenotype. Possible reasons include the overall genetic background, i.e., a complex combination of different genetic variations, or more specific genetic factors with larger detrimental or protective effects. Both phenomena have been described in mouse models, ${ }^{22,23}$ but their role in human epilepsy is not understood.

The remaining 4 mutations that were investigated functionally showed a clear loss of function, including a strong reduction in GABA-evoked current amplitudes and, as demonstrated for p.Y302C and p.Y184H, in GABA sensitivity. This is in line with previous findings in other $G_{A B A_{A}}$ receptor subunits causing similar syndromes. ${ }^{2,4,5,24,25}$ Accordingly, our results suggest GABAergic disinhibition as a major disease mechanism in genetic epilepsies due to pathogenic GABRB3 variants. This is corroborated in a recent study in which 4 EE-associated GABRB3 mutations showed loss of function with a possible genotype-phenotype correlation. ${ }^{26}$ Future studies will clarify whether these findings may extend to a number of novel mutations reported in our publication.

We identified several recurrent mutations and observed a wide range of phenotypic variability for mutation carriers. For example, p.Y302C was observed in 2 patients in this study. Patient 3 had focal epilepsy with onset at 7 months of age, rare seizures, and mild ID. In contrast, patient 16 had intractable EE starting at the age of 17 months, which evolved into LGS with severe ID. The mutation has previously been reported in a patient with onset of focal epilepsy starting at the age of 10 months that 
evolved into LGS with severe ID. Furthermore, p. R232Q was observed in 2 individuals (patients 5 and 6) with moderate ID and treatable multifocal epilepsy with onset at 4 and 11 months of age, respectively. Patient 5 had autistic features and bifrontal heterotopias on MRI, features that were not present in patient 6 , indicating that the phenotypic variability of single mutations may also include the presence of brain malformations. These results indicate a considerable pleiotropy even for single mutations. We were not able to identify a straightforward genotypephenotype correlation, as previously described for other genes associated with genetic epilepsy. ${ }^{15,27}$

Of note, we found 5 patients with a phenotype compatible with MAE, forming a separate subgroup within the spectrum of GABRB3-related phenotypes. A genetic etiology for MAE has been suggested for decades, and several studies have shown that MAE can occur in GEFS+ families with SCN1A or GABRG2 mutations. ${ }^{6,13,28-33}$ Furthermore, mutations in $S L C 2 A 1$ have been found in a subset of patients with MAE and paroxysmal exertional dyskinesia/ motor dysfunction. ${ }^{34,35}$ Recently, we described mutations in SLC6A1, encoding one of the major GABA transporters, in several probands with $\mathrm{MAE}^{36}$; in addition, de novo mutations in GABRG2 and CHD2 have been reported. ${ }^{37}$ The present study adds $G A B R B 3$ to the growing list of genes associated with MAE.

For some of the GABRB3 variants identified in our cohort, the evidence for pathogenicity is limited, and further validating studies are required to understand their significance. This limitation applies to all variants found in familial cases, given that none of the families were large enough to generate significant linkage. In addition, the fact that 2 of the familial variants did not show a significant change in function in Xenopus oocytes further complicates the interpretation. Interestingly, all families contained phenocopies. While this phenomenon is well known in familial fever-related epilepsies, it does not support our current evidence of the pathogenicity of the variants. Two individuals in the ExAC database were found to carry the predicted p.A69T variant, which we detected in our EE group. While there is no clear consensus in the field on how very-low-frequency ExAC mutations should be interpreted, the apparent presence of these variants in presumably unaffected individuals casts some doubt on the role of the variant in the etiology of the patient's disease. Furthermore, 2 truncating variants were inherited from unaffected parents, suggesting that the loss of one allele in these patients either is not associated with the disease or does not show full penetrance. The fact that GABRB3 truncations have not been reported in the ExAC database argues for the latter hypothesis. It has also been shown that the epileptic phenotype observed in mouse models lacking one allele of a $\mathrm{GABA}_{\mathrm{A}}$ receptor subunit gene may be milder compared to missense mutation carriers, ${ }^{38}$ although this may depend on the functional interactions of the truncated protein and the remaining WT subunits. ${ }^{39}$ Lastly, we cannot completely exclude that the parents carry these mutations in a mosaic state, as seen in the mother of patient 9, even though this is not obvious from the blood DNA analysis. ${ }^{40}$

This study demonstrates that GABRB3 mutations are associated with a phenotypic spectrum ranging from simple FSs, GEFS +, and EOAE to MAE, EE within the DS spectrum, and other severe EE, defining a novel genetic entity within the GEFS+ and EOEE spectrum. GABRB3 mutations cause reduced receptor function, predicting impairment of GABAmediated inhibition as disease mechanism.

\section{AUTHOR CONTRIBUTIONS}

R.S.M. and T.V.W. conceived and designed the study, collected and analyzed data, and wrote the manuscript. I.H. collected and analyzed data and edited the manuscript. C.M. provided inclusion of patients and analysis of phenotypic data. K.M.J. collected and analyzed data. E.H.B,.U.V., I.B., I.T., T.T., G.K., and L.L.F. provided inclusion of patients and analysis of phenotypic data. G.L. and J.d.B. collected and analyzed data. S.B. provided inclusion of patients and analysis of phenotypic data. N.C. collected and analyzed data. N.H., J.J., M.S., C.B., S.S., and M.N. provided inclusion of patients and analysis of phenotypic data. C.T.M., L.H.G.L., S.V., and M.P. collected and analyzed data. S.v.S., S.H., and H.D. provided inclusion of patients and analysis of phenotypic data. Y.M. collected and analyzed data. K.M., H.H., and K.L.v.G. provided inclusion of patients and analysis of phenotypic data. H.A.D., N.T., H.C.M., and G.R. collected and analyzed data. R.G. provided inclusion of patients and analysis of phenotypic data. J.R.L. collected and analyzed data. H.L. collected and analyzed data and edited the manuscript. H.M. and S.M. conceived and designed the study, collected and analyzed data, and edited the manuscript.

\section{ACKNOWLEDGMENT}

We thank the patients and their families for participating in this study. We also thank Nicole Zepezauer and Carolin Schilpp for their excellent technical assistance and Dr. Ulrike Hedrich, Dr. Cristina Niturad, and Merle Harrer for helpful discussion and assistance.

\section{STUDY FUNDING}

This study was realized with funding from the Lundbeck Foundation (2013-14290), the University of Copenhagen Program for Interdisciplinary Research (Global Genes, Local Concerns), the Danish Council for Independent Research- Medical Sciences (4183-00), the EuroEPINOMICS grant SARLA 11091E, EuroEPINOMICS grant Le1030/11-2, BMBF grant IonNeurONet 01 GM1105A, and the European Union Seventh Framework Program FP7/2007-2013 under the project DESIRE (grant agreement 602531).

\section{DISCLOSURE}

R. Møller, T. Wuttke, and I. Helbig report no disclosures relevant to the manuscript. C. Marini reports being associate editor of epileptic disorders; receiving speaker honoraria at VII Congresso Nazionale Simmesn-Firenze, December 16-18, 2015, from the Swedish Orphan Biovitrum; serving as a member of the editorial board of Epilepsia (2007-2012); being associate editor of epileptic disorders (2014), Italian Minister of Health, Research Program Section (RF- 2009-1525669; title of the project: Clinical and Genetic Study of Early Onset Epilepsies of Unknown Aetiology; duration, 2011-2013); and receiving a research grant from CARIPLO Foundation, 
Milan, Italy (January 5, 2014-January 2, 2016): Clinical Characterization of PCDH19 Mutations. K. Johannesen, E. Brilstra, U. Vaher, I. Borggraefe, I. Talvik, T. Talvik, G. Kluger, L. Francois, G. Lesca, J. de Bellescize, S. Blichfeldt, N. Chatron, N. Holert, J. Jacobs, M. Swinkels, C. Betzler, S. Syrbe, M. Nikanorova, and C. Myers report no disclosures relevant to the manuscript. L. Larsen is employed in a commercial genetic testing company, Amplexa Genetics. S. Vejzovic, M. Pendziwiat, S. von Spiczak, S. Hopkins, H. Dubbs, Y. Mang, K. Mukhin, H. Holthausen, and K. van Gassen report no disclosures relevant to the manuscript. H. Dahl is employed in a commercial genetic testing company, Amplexa Genetics. N. Tommerup, H. Mefford, G. Rubboli, R. Guerrini, and J. Lemke report no disclosures relevant to the manuscript. H. Lerche reports having received travel support or honoraries for speaking or consulting from the following pharmaceutical companies: Bial, Desitin, Esai, Pfizer, and UCB. H. Muhle and S. Maljevic report no disclosures relevant to the manuscript. Go to Neurology.org for full disclosures.

Received May 12, 2016. Accepted in final form November 7, 2016.

\section{REFERENCES}

1. Macdonald RL, Kang J, Gallagher MJ. GABA $A_{A}$ receptor subunit mutations, and genetic epilepsies. In: Noebels JL, Avoli M, Rogawski MA, Olsen RW, Delgado-Escueta AV, editors. Jasper's Basic Mechanisms of the Epilepsies, 4th ed. Bethesda: National Center for Biotechnology Information; 2012.

2. Baulac S, Huberfeld G, Gourfinkel-An I, et al. First genetic evidence of GABA(A) receptor dysfunction in epilepsy: a mutation in the gamma2-subunit gene. Nat Genet 2001;28:46-48

3. Kananura C, Haug K, Sander T, et al. A splice-site mutation in GABRG2 associated with childhood absence epilepsy and febrile convulsions. Arch Neurol 2002;59:1137-1141.

4. Maljevic S, Krampfl K, Cobilanschi J, et al. A mutation in the $\mathrm{GABA}(\mathrm{A})$ receptor alpha(1)-subunit is associated with absence epilepsy. Ann Neurol 2006;59:983-987.

5. Cossette P, Liu L, Brisebois K, et al. Mutation of GABRA1 in an autosomal dominant form of juvenile myoclonic epilepsy. Nat Genet 2002;31:184-189.

6. Wallace RH, Wang DW, Singh R, et al. Febrile seizures and generalized epilepsy associated with a mutation in the $\mathrm{Na}+$-channel betal subunit gene SCN1B. Nat Genet 1998;19:366-370.

7. Allen AS, Berkovic SF, Cossette P, et al. De novo mutations in epileptic encephalopathies. Nature 2013;501:217-221.

8. Carvill GL, Weckhuysen S, McMahon JM, et al. GABRA1 and STXBP1: novel genetic causes of Dravet syndrome. Neurology 2014;82:1245-1253.

9. De novo mutations in SLC1A2 and CACNA1A are important causes of epileptic encephalopathies. Am J Hum Genet 2016;99:287-298.

10. Johannesen K, Marini C, Pfeffer S, et al. Phenotypic spectrum of GABRA1: from generalized epilepsies to severe epileptic encephalopathies. Neurology 2016;87: 1140-1151.

11. Zhang Y, Kong W, Gao Y, et al. Gene mutation analysis in 253 Chinese children with unexplained epilepsy and intellectual/developmental disabilities. PLoS One 2015; 10:e0141782.

12. Hamdan FF, Srour M, Capo-Chichi JM, et al. De novo mutations in moderate or severe intellectual disability. PLoS Genet 2014;10:e1004772.

13. Doose H, Baier WK. Epilepsy with primarily generalized myoclonic-astatic seizures: a genetically determined disease. Eur J Pediatr 1987;146:550-554.
14. Papandreou A, McTague A, Trump N, et al. GABRB3 mutations: a new and emerging cause of early infantile epileptic encephalopathy. Dev Med Child Neurol 2015; 58:416-420.

15. McTague A, Howell KB, Cross JH, Kurian MA, Scheffer IE. The genetic landscape of the epileptic encephalopathies of infancy and childhood. Lancet Neurol 2016;15:304-316.

16. Berg AT, Berkovic SF, Brodie MJ, et al. Revised terminology and concepts for organization of seizures and epilepsies: report of the ILAE Commission on Classification and Terminology, 2005-2009. Epilepsia 2010;51:676-685.

17. Orhan G, Bock M, Schepers D, et al. Dominant-negative effects of KCNQ2 mutations are associated with epileptic encephalopathy. Ann Neurol 2014;75:382-394.

18. Møller RS, Larsen LH, Johannesen KM, et al. Gene panel testing in epileptic encephalopathies and familial epilepsies. Mol Syndromol 2016;7:210-219.

19. Wen M, Yan Y, Yan N, Chen XS, Liu SY, Feng ZH. Upregulation of RBFOX1 in the malformed cortex of patients with intractable epilepsy and in cultured rat neurons. Int J Mol Med 2015;35:597-606.

20. Goldberg-Stern H, Aharoni S, Afawi Z, et al. Broad phenotypic heterogeneity due to a novel SCN1A mutation in a family with genetic epilepsy with febrile seizures plus. J Child Neurol 2014;29:221-226.

21. Johnston AJ, Kang JQ, Shen W, et al. A novel GABRG2 mutation, p.R136*, in a family with GEFS+ and extended phenotypes. Neurobiol Dis 2014;64:131-141.

22. Rubinstein M, Westenbroek RE, Yu FH, Jones CJ, Scheuer T, Catterall WA. Genetic background modulates impaired excitability of inhibitory neurons in a mouse model of Dravet syndrome. Neurobiol Dis 2015;73:106-117.

23. Miller AR, Hawkins NA, McCollom CE, Kearney JA. Mapping genetic modifiers of survival in a mouse model of Dravet syndrome. Genes Brain Behav 2014;13:163-172.

24. Kang JQ, Macdonald RL. The GABAA receptor gamma2 subunit R43Q mutation linked to childhood absence epilepsy and febrile seizures causes retention of alpha1beta2gamma2S receptors in the endoplasmic reticulum. J Neurosci 2004;24: 8672-8677.

25. Hirose S. Mutant GABA(A) receptor subunits in genetic (idiopathic) epilepsy. Prog Brain Res 2014;213:55-85.

26. Janve VS, Hernandez CC, Verdier KM, Hu N, Macdonald RL. Epileptic encephalopathy de novo GABRB mutations impair GABA receptor function. Ann Neurol Epub 2016 Mar 7.

27. Moller RS, Heron SE, Larsen LH, et al. Mutations in KCNT1 cause a spectrum of focal epilepsies. Epilepsia 2015;56:e114-e120.

28. Doose H, Gerken H, Leonhardt R, Volzke E, Volz C. Centrencephalic myoclonic-astatic petit mal: clinical and genetic investigation. Neuropadiatrie 1970;2:59-78.

29. Wallace RH, Marini C, Petrou S, et al. Mutant GABA(A) receptor gamma2-subunit in childhood absence epilepsy and febrile seizures. Nat Genet 2001;28:49-52.

30. Wallace RH, Scheffer IE, Barnett S, et al. Neuronal sodium-channel alpha1-subunit mutations in generalized epilepsy with febrile seizures plus. Am J Hum Genet 2001; 68:859-865.

31. Dimova PS, Yordanova I, Bojinova V, Jordanova A, Kremenski I. Generalized epilepsy with febrile seizures plus: novel SCN1A mutation. Pediatr Neurol 2010;42:137-140.

32. Yordanova I, Todorov $\mathrm{T}$, Dimova $\mathrm{P}$, et al. One novel Dravet syndrome causing mutation and one recurrent 
MAE causing mutation in SCN1A gene. Neurosci Lett 2011;494:180-183.

33. Escayg A, Heils A, MacDonald BT, Haug K, Sander T, Meisler MH. A novel SCN1A mutation associated with generalized epilepsy with febrile seizures plus-and prevalence of variants in patients with epilepsy. Am J Hum Genet 2001;68:866-873.

34. Mullen SA, Marini C, Suls A, et al. Glucose transporter 1 deficiency as a treatable cause of myoclonic astatic epilepsy. ArchNeurol 2011;68:1152-1155.

35. Larsen J, Johannesen KM, Ek J, et al. The role of SLC2A1 mutations in myoclonic astatic epilepsy and absence epilepsy, and the estimated frequency of GLUT1 deficiency syndrome. Epilepsia 2015;56: e203-e208.

36. Carvill GL, McMahon JM, Schneider A, et al. Mutations in the GABA transporter SLC6A1 cause epilepsy with myoclonic-atonic seizures. Am J Hum Genet 2015;96: 808-815.

37. Carvill GL, Heavin SB, Yendle SC, et al. Targeted resequencing in epileptic encephalopathies identifies de novo mutations in CHD2 and SYNGAP1. Nat Genet 2013;45: 825-830.

38. Reid CA, Kim T, Phillips AM, et al. Multiple molecular mechanisms for a single GABAA mutation in epilepsy. Neurology 2013;80:1003-1008.

39. Warner TA, Shen W, Huang X, Liu Z, Macdonald RL, Kang JQ. Differential molecular and behavioural alterations in mouse models of GABRG2 haploinsufficiency versus dominant negative mutations associated with human epilepsy. Hum Mol Genet Epub 2016 Jun 13.

40. Milh M, Lacoste C, Cacciagli P, et al. Variable clinical expression in patients with mosaicism for KCNQ2 mutations. Am J Med Genet A 2015;167A:2314-2318.

\section{Save These Dates for AAN CME Opportunities!}

Mark these dates on your calendar for exciting continuing education conferences by the American Academy of Neurology. Learn more at AAN.com/conferences.

\section{AAN Annual Meeting}

- April 22-28, 2017, Boston, MA, at the Boston Convention Center 